\title{
Henry Heller, The Bourgeois Révolution in France,
} 1789-1815

New York, Bergham Books, 2006, 184 p., ISBN 1-84545-169-4, 60 \$.

Julien Louvrier

\section{(2) OpenEdition}

\section{Journals}

Édition électronique

URL : https://journals.openedition.org/ahrf/11414

DOI : 10.4000/ahrf.11414

ISSN : 1952-403X

Éditeur :

Armand Colin, Société des études robespierristes

Édition imprimée

Date de publication : 1 mars 2008

Pagination : 218-221

ISSN : 0003-4436

Référence électronique

Julien Louvrier, « Henry Heller, The Bourgeois Révolution in France, 1789-1815», Annales historiques de la Révolution française [En ligne], 351 | janvier-mars 2008, mis en ligne le 29 décembre 2009, consulté le 06 août 2021. URL : http://journals.openedition.org/ahrf/11414 ; DOI : https://doi.org/10.4000/ahrf. 11414 
n'a donné suite qu'en France, où la rupture sociale a permis la reconnaissance en tant que telle de l'idéologie des droits de l'homme. Mais en retour, l'état même des relations entre les groupes confessionnels, ethniques et sexués n'a autorisé leur application qu'aux minorités protestantes, et juives avec retard, aux esclaves temporairement et jamais aux femmes. La conjonction entre idéologie et luttes politiques explique le lent délai pour leur établissement après 1948. Ce livre noue ainsi de façon tout à fait convaincante l'histoire culturelle «à l'américaine ", est-on tenté de dire pour souligner que ces mots renvoient à la prise en considération de toutes les trames qui organisent une société, avec l'histoire politique événementiclle, pour déboucher ainsi sur la résolution des questions régulièrement évoquées autour de l'unité et de la discontinuité des phénomènes historiques, autour de l'articulation entre temps court et temps long, entre idéologie et pratique, mais aussi entre les versants lumineux et sombres d'un même objet historique. Lynn Hunt n'évite pas les apories des droits de l'homme, pas plus que les conséquences inséparables de leur application dans l'établissement d'un universalisme pouvant être démobilisateur et d'un accroissement de l'individualisme. Mais c'est sur leur effet performatif qu'elle conclut, soulignant que si les droits ne résolvent pas tous les problèmes qui se posent, leur proclamation oblige cependant à les affronter, donnant ainsi la valeur à ces morceaux de papiers qui ne sont décidemment pas des pensées sans efficacité.

Jean-Clément MARTIN

Henry Heller, The Bourgeois Revolution in France, 1789-1815, New York, Bergham Books, 2006, 184 p., ISBN 1-84545-169-4, $60 \$$.

Dans la courte préface introductive à son histoire de la « Révolution bourgeoise ", Henri Heller, professeur à l'Université de Manitoba au Canada, spécialiste de la France du XVI' siècle, annonce sans ambages l'objectif assigné à ce nouveau livre : "Réaffirmer le caractère bourgeois ct capitaliste de la Révolution française ", suivant en cela la vision de Marx, elle-méme influencée par les historiens libéraux de la Restauration. Ce faisant, l'auteur entend rendre visibilité et légitimité à une interprétation marxiste de la Révolution maltraitée par plus de trente années de révisionnismes tous azimuts.

Élaborée sur la base de nombreuses lectures - principalement de travaux français récents consacrés à l'histoire économique de la Révolution auxquels l'auteur emprunte à la fois les données empiriques et les analyses - la réponse d'Henri Heller à l'historiographie " révisionniste " s'articule en trois points. Tout d'abord, il s'agit d'interroger de façon critique le bilan des révisionnismes au regard des perspectives ouvertes par les travaux d'inspiration marxistc publićs ces vingt dernières années. Pour Henri Heller, la vague révisionniste qui a frappé I'historiographie de la Révolution française trouve ses origines dans la réaction antimarxiste - à la fois idéologique et académique - des années 1970. Malgré leur grande diversité, c'est bien dans leur opposition déclarée au contenu marxiste de l'interprétation classique de la Révolution française - personnifiée, d'après l'auteur, par Albert Mathiez, Georges Lefebvre, Alhert Soboul et Michel Vovelle - que les révisionnismes ont trouvé un terrain d'entente : que l'on pense à la « théorie des élites » de Denis Richet, à la « richesse non-capitaliste " de l'Américain Georges Taylor, ou 
plus récemment encore, à l'étude de Sarah Maza sur la bourgeoisie (The Myth of the French Bourgeoisie: An Essay on Social Imaginary, 1750-1850, 2003) qui refuse toute existence à la bourgeoisie française tant que le discours des bourgeois n'atteste pas en mots d'une conscience de classe en fait. Ces hypothèses de recherche sont toutes dirigées contre l'interprétation qui voit dans la dynamique révolutionnaire de 17891799 le résultat du développement historique d'une classe bourgeoise consciente d'elle-même. Tandis que de très nombreux travaux d'histoire économique ou socioculturelle sont venus dernièrement étayer la thèse de la révolution bourgeoise et soutenir l'importance du développement capitaliste dans le déclenchement de la Révolution française, Heller fait observer que dans le monde anglo-saxon la vision " critique " domine sans partage. D'où la formule choisic par l'auteur qui consiste à bâtir son argumentation sur des travaux français non traduits et peu accessibles au public non-francophone, une façon de diffuser au-delà des cercles de spécialistes les éléments manquants du débat.

Dans un second temps, qui est le cœur de l'ouvrage, Heller entend démontrer que l'économie française disposait à la fin du XVIII' siècle d'un certain nombre d'atouts en faveur d'un véritable développement capitaliste, sinon dans la mesure de l'économie anglaise, du moins sur ses traces. Attachée à suivre l'évolution des structures économiques de la France pré-révolutionnaire, tant du point de vue de l'agriculture que du côté du commerce et des manufactures, son analyse donne lieu à deux chapitres fort documentés. Examinant les transformations progressives des campagnes, l'apparition des grandes fermes dans le Nord, la croissance du recours au travail salarié, la naissance d'une bourgeoisic rurale d'Ancien Régime dont les revenus intègrent de plus en plus de profits capitalistes à côté des rentes féodales, Heller accumule tout un faisceau de preuves historiquement constituées qui attestent la vitalité de l'économie française à la veille de la Révolution et surtout le scnsible développement d'une économie de type capitaliste qui trouve dans la paysannerie une main-d'ouvre bon marché et de plus en plus dépendante du travail salarié. Lémergence du capitalisme est néanmoins décrite avec certaines prćcautions. L'auteur ne dissimule pas les résistances qui freinent ou limitent son développement, résistance populaire face à l'innovation technologique par peur du déclassement (bris de machines), résistance intellectuelle des élites savantes par dédain pour les sciences pratiques (les difficultés de Vaucanson à l'Académie). Ainsi, il n'hésite pas à conclure que si l'Angleterre du XVIII ${ }^{\circledR}$ siècle était une société complètement capitaliste, tel n'était pas le cas de la France.

En faisant la synthèse des multiples signes précurseurs d'un développement capitaliste de l'économie française à la fin du XVIII' siècle, l'historien canadien ne fait pas que batailler avec les révisionnistes patentés, il entreprend ćgalement de rouvrir le débat avec leurs homologues marxistes anglo-saxons pour lesquels la Révolution française n'a pu être bourgeoise et encore moins capitaliste dans la mesure où la paysannerie de 1789 était encore loin d'avoir été complètement "prolćtarianisée ", i.e. renduc exclusivement dépendante du marché pour subsister. Emmenés par l'historien américain Robert Brenner, les animateurs de cette école de pensée d'inspiration althussérienne et dont les thèses ont été appliquées à la Révolution française par le canadien George Comninel, considèrent que l'on ne peut parler de Révolution bourgeoise en France dans la mesure où malgré les nouveaux rapports de production induits par le développement de l'industrie dans les interstices du système « féodal », l'absolutisme français n'avait aucune des carac- 
téristiques d'une économie capitaliste. À l'opposé d'une vision stricte des mécanismes économiques qui permettent de démontrer l'existence d'une organisation sociale authentiquement capitaliste, Heller souligne au contraire l'importance qu'il faut accorder aux formes intermédiaires de travail et de production dans une société en pleine transition. Ce livre s'inscrit donc en réalité dans un débat très relevé qui agite depuis longtemps la communauté scientifique marxiste autour de la nature de la révolution bourgeoise et du lien intime qui l'unit au développement du capitalisme. Depuis la controverse fondatrice qui opposa en leur temps les économistes Maurice Dobb et Paul Sweezy, le débat sur les formes de la transition au capitalisme ne s'est pas relâché. On sait, grâce à l'intelligente publication chez Maspéro au début des années 1970 des principaux éléments du débat, combien cette question préoccupa les spécialistes de nombreux pays, de Georges Lefebvre à Éric Hobsbawm en passant par le Japonais Takahashi. Il faut désormais compter avec les arguments rassemblés par Henri Heller.

En dernier lieu, l'auteur se propose d'examiner de façon chronologique les différents axes constituant la politique économique mise en place par les Assemblées révolutionnaires successives jusqu'à la prise en main des affaires par le général devenu empereur. Le travail de la Législative, marqué par de célèbres mesures en faveur du libéralisme économique, est apprécié à travers les exemples de Paris et de Lille. Heller montre combien la Révolution, loin de produire une nouvelle bourgeoisie, s'est appuyée sur des groupes préexistants. Dans la grande ville du Nord, ce sont ces milieux d'affaires qui, s'étant emparés des commandes de l'administration locale, ont bénéficié du soutien de l'Assemblée pour faire redémarrer manufactures et industries. L'analyse de la période de la Convention, qui fait une place importante aux travaux de l'historien soviétique Anatoli Ado - ce qui permet tout de même à Heller de faire remarquer que l'historiographie marxiste a connu elle aussi des retournements - est tout aussi stimulante. En revanche, le chapitre consacré au Directoire, s'il accorde à la période une importance dont la tradition historiographique l'avait longtemps privé, est tout entièrement fondé sur les thèses de James Livesey dont J.-P. Jessenne a pourtant montré les limites (voir son compte rendu, $R H M C, 2004 / 2$ ). Pour finir, l'analyse de l'ère napoléonienne est somme toute classique. En insistant sur la relative continuité avec les tendances économiques qui avaient caractérisé les périodes révolutionnaires précédentes, lauteur reprend à son compte les précicuses indications fournies par François Hincker dans le Dictionnaire historique de la Révolution française.

Si le livre a une réelle utilité dans le contexte anglophone où la sphère académique est dominée par les travaux non-marxistes voire anti-marxistes, du côté français, il risque en revanche d'irriter les chercheurs par sa rhétorique souvent militante et parfois peu convaincante. Toutefois, le défi que s'est lancé Heller est intéressant et constitue un utile contrepoids dans un moment où penser avec Marx semble avoir disparu de l'agenda scientifique. De ce point de vue, l'auteur fournit un véritable panorama des efforts entrepris en ce sens en France et démontre la vitalité d'unc tradition d'étude, certes contestée et critiquée, mais bien vivante et surtout en constant renouvellement. On l'aura compris, l'intérêt de ce livre ne réside pas tant dans les données empiriques qu'il rassemble que dans sa tentative pour faire entendre une autre voix que celle d'une historiographie anglo-saxonne qui a succombé aux théories de la culture et à l'analyse du discours, au profit de travaux qui confèrent à l'instance économique et sociale une place capitale dans 
l'explication du développement historique. Toutefois, si l'entreprise est courageuse, elle n'est pas sans susciter quelques remarques, notamment du point de vue de la terminologie. Lauteur a largement recours à ces riches notions que sont justement les expressions " révolution bourgeoise ", "lutte des classes " et " bourgeoisie », mais qui malheureusement ne font l'objet d'aucune explication en profondeur permettant de retracer leur généalogie, leur sens chez Marx et leur sens dans le discours historiographique sur la Révolution française. Par ailleurs, Henri Heller a visiblement négligé la dimension internationale de la Révolution française, y compris dans son analyse économique qui semble ignorer les problématiques coloniales. Mais ce ne sont là que détails qui ne doivent pas nous faire oublier qu'en remettant au centre de la réflexion historiographique des thèmes et des questions aussi importants que le rapport entre révolution et capitalisme, Henri Heller nous offre des perspectives de recherche renouvelées.

Julien LOUVRIER

James M. Anderson, Daily Life During the French Revolution, Westport (Connecticut) et Londres, Greenwood Press, 2007, 269 p., ISBN-13: 978-0-31333683-6, 130,95\$.

Déjà auteur d'une histoire du Portugal (2000) et d'une vie quotidienne pendant l'Inquisition espagnole (2002), ce professeur émérite de l'Université de Calgary au Canada n'est pas à son premier coup d'essai en matière d'histoire sociale. Sans être lui-même spécialiste de l'histoire de France, soulignons que James Anderson, en composant sa fresque, a eu recours aux travaux les plus récents d'historiens de la période révolutionnaire : qu'il suffise de mentionner les noms de Colin Jones, Christine Hillam ou John Frangos, pour ce qui est de l'histoire de la médecine et des hôpitaux, ou encore ceux de Gwynne Lewis, John Lough et David Garrioch, en ce qui concerne l'histoire sociale proprement dite. De manière plus générale, il a su intégrer dans son tableau l'éclairage un tant soit peu contestataire de Lynn Hunt, Suzanne Desan ou David Andress. Louvrage de James Anderson est par conséquent solidement documenté.

Comme on le sait, les récits de la "vie quotidienne " constituent un genre reconnu et consacré de l'historiographic anglo-saxonne, s'inscrivant dans la lignée de l'ouvrage classique de G. M. Trevelyan, English Social History (première édition parue en 1944). Genre souvent exploité avec panache en France même, qu'il suffise d'évoquer le chef-d'œuvre du regretté Henri Amouroux, La grande histoire des Français sous l'occupation. Pour ce qui est de 1789, l'ouvrage déjà ancien de Jean Robiquet sur la vie quotidienne sous la Révolution méritait une mise à jour, Anderson s'en est inspiré, en y apportant des compléments très riches issus de la recherche moderne. Il n'en reste pas moins que l'auteur se trouve confronté à une véritable gageure, en raison même de son approche du sujet. Face à une Révolution française faite d'incessants rebondissements, dont il ne ressort a priori aucune trame ćvolutive cohérente, et dont l'incidence immédiatc sur la vie des citoyens n'est pas aisée à déterminer, Anderson s'efforce de relever un triple défi : la nécessité de nous raconter l'Ancien Régime, afin de nous faire comprendre ce que la Révolution a balayé, puis de nous parler de la suite ; car, comme il l'affirme dans sa 